\title{
Online communities as a source of innovation: A netnographic study on Crusader Kings 3
}

\author{
Kevin Walther \\ Uppsala University \\ Kevin.walther@,fek.uu.se
}

\begin{abstract}
Video Game development is driven more and more by the input from player communities - both prior to release and post-release. This study focuses on a particular game community using a netnographic approach and applies the theoretical lens of free innovation. Data stems from analyzing 1,798 forum threads based on players' ideas and suggestions, 9 interviews with active forum contributors and 2 community managers. NVivo software was used to code the suggestion threads into themes. Subsequently, the themes were analyzed based on the extent to which they were implemented into the game within the timeframe of the first four months postrelease. This study thus sheds light on community management in the video games industry and players spending their free time on idea generation. Moreover, through thematic coding, the article offers a potential method on how to converge the vast amount of qualitative data stemming from player communities' suggestions.
\end{abstract}

\section{Introduction}

The video game industry provides an interesting context for examining user innovation and communities. Take "Defense of the Ancients 2" (better known as Dota 2) from the American game "Valve" as an example. The first version of DOTA was developed by a user with the Pseudonym "Eul" as a free modification to the game Warcraft 3. Eul released the modification in 2003, and soon the modification became more popular than the basic version of Warcraft 3. Fast forwarding, the American firm and giant in the video game industry Valve acquired the intellectual property rights to Dota in 2009 and hired Eul and other people behind the Dota modification. Dota 2 was eventually released in 2013 and became one of the most successful games in video game history. The game is available on Valve's digital distribution platform "Steam," and still in January 2021 , the game has almost 600,000 active monthly players [26].
The Dota modification is a prime example of consumer innovation. In many industries, consumer innovations are thriving, thanks to the easy access to free information and the internet - where innovative users can easily connect and collaborate $[12,13]$. This is, of course, not a new phenomenon. Earlier research points out that customer-driven companies do well to exploit their capabilities to leverage on the knowledge of their customers [10]. Video games are easily modifiable by their players and can in an extreme case lead to a success story like Dota 2 . This indicates the importance of game developers following up on ideas and modifications stemming from their community. The consumers/ players rarely benefit financially from their suggestions, and the innovations are developed at a private cost in their free time. In most cases, a modification or idea is not protected by its creators, so the innovation can in theory be taken on by anyone without cost. Thus, a vast amount of video game consumers' ideas and innovations can be categorized as free innovations. From a firm perspective, integrating free innovation into a corporate innovation pipeline provides a valuable resource [13]. Various studies in contexts ranging from toys to sports and to banking services have examined how firms implement consumer innovations $[1,8,18]$. Furthermore, studies conducted in the context of the software or video game industry highlighted the value of user generated content [14], unpaid crowd complementors [4], or encouraging users to innovate through software toolkits [20].

This research focuses on one particular player community and development firm. The aim is to gain an understanding of how a digital product is altered post-release, based on the suggestions and ideas from its consumer base. This study emphasizes the actual players' suggestions on how a game can be incrementally improved post-release rather than actual user generated content. The case firm selected for this study is the Swedish video game developer and publisher Paradox Interactive. More specifically, their latest game release Crusader Kings 3 (CK3) is the subject of this study. The firm is known for complex historically-themed strategy games. Paradox Interactive also actively supports user generated 
content and community culture through various channels such as moderated online forums and discord servers, where players can directly communicate with community managers. A netnographic approach was chosen to collect data from the official Paradox Interactive online forum. Additionally, written interviews were conducted with highly active contributors to the official forum and community managers. The suggestions made by the players were captured with NVivo and coded into themes. Each theme was then analyzed through NVivo's word query feature and compared with the patch notes of the examined game. This approach allowed us to analyze whether the suggestions and thus free innovations provided by users were addressed in the updated version of the game.

The outline of the article is as follows: in the first section, the concept of free innovation is explained, and parallels from von Hippel's [13] free innovation paradigm are made with the help of examples from the video game industry. In the method section, the netnographic method of this study is explained, and Paradox Interactive \& CK3 are briefly introduced. In the findings, results are presented with the help of data analysis made with NVivo. The paper ends with a discussion on the free innovation paradigm in the video game industry.

\section{Free Innovation}

In classic economic theory, household sector consumers have been traditionally viewed as passive users of producer-developed products and services. Consumers do not produce themselves and affect the economy only by consumption. Or as stated by Schumpeter (1934): "It is, however, the producer who as a rule initiates economic change, and consumers are educated by him if necessary; they are, as it were, taught to want new things, or things which differ in some respect or other from those which they have been in the habit of using."

Recent research, however, challenges this traditional Schumpeterian view and finds that consumers develop a lot of innovation too. Collaborative creation, interactivity, and community culture are concepts that have been converged by firms into creating consumer engagement and value [25, 28]. A study by Bengtsson [2] e.g. shows that $7.3 \%$ of Swedish consumers are innovating consumers. It can be assumed therefore that a lot of innovation is rooted in consumer innovations and thus stemming from the traditionally described household sector. A large part of consumer innovation is motivated by self-rewards, and the innovations are developed during unpaid, discretionary time. Moreover, consumers seldom protect their innovations, and this consequently does not prevent others from adopting them. Including the above-cited country-based studies and based on surveys conducted in different countries, von Hippel [12] estimates that $90 \%$ of consumer innovations are free innovations, where consumer innovators are not paid in any form for their innovation expenditures. On the other hand, producers' R\&D departments are paid and have to justify investments in innovation.

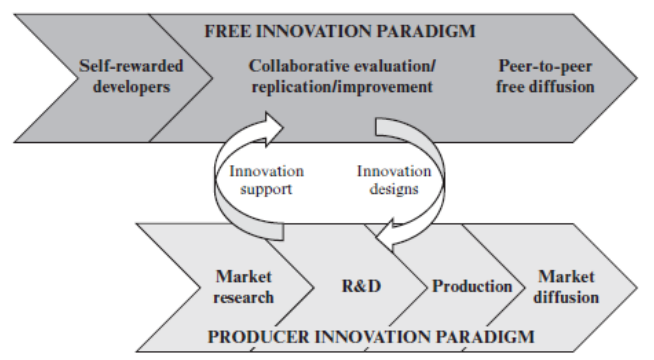

Figure 1. Free innovation paradigm by von Hippel

Following von Hippel's [12] argumentation, this calls for a new paradigm beyond the Schumpeterian "producer innovation paradigm," as the free innovation paradigm cannot be incorporated into the producer innovation paradigm. Figure 1 shows how the two paradigms are different but de facto, the free innovation paradigm can be utilized by producers for their R\&D processes. Free innovation is distributed without financial benefit among peers, whereas producers seek market diffusion for profit. Nevertheless, it can be desirable for a producer to challenge the producer innovation paradigm and seek innovation through collaboration with free innovators. Based on different studies on free innovation $[1,8,18]$, von Hippel (2017) identifies five different distinct ways for how producers can collaborate with and benefit from free innovation. I describe these five recommendations hereafter by giving examples from the video game industry.

\section{Directing free innovator effort to particular areas} or approaches through tools and other support

The first recommendation by von Hippel is to give tools and other types of support to the consumers/ community. The most prominent example is Valve, who offers its platform users a secondary platform called Steam Workshop - where users can create, discover, and download content for their games for free [27]. According to von Hippel [13], producers can guide free innovators in directions that are profitable to them and create a flow of content for their products. Also, for owners of digital marketplaces or game platforms, free innovators are beneficial. A study by Boudreau and Jeppsen [4], with empirical data from 
85 game platforms, finds that unpaid complementors respond to the growth of game platforms.

\section{Learning from free innovator pioneering}

A study by Ho-Dac [14], which is based on empirical data from 1,287 software projects, shows how user innovation outside the firm has positive effects on the initiation and completion of product development activities. In line with that is von Hippel's second recommendation, which pinpoints how producers can profit from free innovation by considering free innovation as pioneering work. Referring to the example from the introduction, the initial Dota modification established an entirely new genre of Multiplayer Online Battle Arena (MOBA). MOBA is a subgenre of strategy games, where players compete against each other on a predefined battlefield and control a single character with a set of unique abilities [5]. Up until the year 2020, two out of the five most popular e-sports games fell under the MOBA genre - one of them being DOTA 2 [22]. The innovators behind the DOTA modification thus pioneered a whole new genre in their free time that the gaming industry thrives upon.

3. Supporting free innovator development of complements to commercial products

Thirdly, producers can support free innovators' development of complements to their commercial products. In the video game context, producers should build a relationship with free innovators who create additional content for the game or are writing down their ideas for future updates or content. By offering discussion forums and interactive social media channels (such as Discord), producers can thus identify players' needs early on and have a dialogue with players that modify the game. Research conducted on various cases from the video game industry points out that user communities can be seen as a locus of innovation and the importance of firms to capture innovative contributions [20,21].

\section{Using competition from free designs to spur} improvements to commercial products

Fourthly, producers should use competition from free designs to stimulate improvements to their commercial products. Producers can of course also see what free innovators produce for competitors' titles. If a certain modification is vastly successful for a competitor's game, a game producer can implement a similar modification into their commercial product. It is easier to purchase a commercial product from a producer rather than searching, downloading, and installing modifications. This typically helps producers to compete with free innovations.
5. Accessing free innovator product designs to reduce in-house development costs

Fifthly, producers should incorporate concepts from free innovators to minimize in-house development costs. In the video games context, this can be both conceptual ideas or technological improvements or debugging. Many video game producers have a bug report in their games so players can report game crashes and errors to the developer. Moreover, Beta tests are common in this industry where a limited number of players are granted access to play the game before release. During these Beta tests, the players are surveyed on their game experience and asked to report potential bugs. This minimizes the effort and cost for the producers to test the game internally, especially as the players are transformed from consumers to testers without any financial incentives, apart from getting a sneak peek of the game pre-release. The success story of the game "Fortnite" shows how important these insights can be from a conceptual level. In its initial version, Fortnite was a so-called tower-defense game where the players must defend his/her base against incoming waves of zombies. The Beta-test however revealed that players mostly ignored this mode but were extremely keen on playing the "battle royale" game mode. The producer "Epic Games" thus completely scrapped the towerdefense aspect of the game after this Beta-test and focused on the battle royale game mode. The game also changed its name from "Fortnite: Save the World" to "Fortnite Battle Royale." Eventually, the game became one of the video game industry's most successful game titles [7].

As these various examples from the video game industry highlight, von Hippel's recommendations can serve as a guideline for a producer to profit from free innovations stemming from enthusiastic individual players and communities. The producer, however, must face the challenging task of how to filter for interesting user innovations that can be diffused into the market and generate a profit. This gets even more complex, especially as innovation in the video game industry is not necessarily a single idea or modification but rather deriving from a dialogue with the user base or something that is trending among consumers. The rest of the article investigates the case of Paradox Interactive that does both - entertain a constant dialogue with its user base and provide tools to modify the base game.

\section{Case Firm}

For this study, the Swedish game publisher and developer "Paradox Interactive" is chosen. More 
specifically, the community members committed to the suggestion forum for their latest title "Crusader Kings 3" (CK3) - released on September 1st, 2020 for the PC platform.

CK3 is a highly complex and fascinating historical strategy game. The overall aim of the game is to lead and manage a family dynasty set in the middle ages. The game offers two starting dates either 867 or 1066 - and ends either with the passing of the last member of the family dynasty or eventually with the fall of the Byzantine Empire in 1459. Some central gameplay aspects of CK3 are intriguing, dynastic feuds, declaring wars, setting up marriage alliances, managing vassals and much more. Each playthrough creates its dynamic and story. One playthrough can take dozens of hours of playtime if the dynasty advances well. Eventually, a playthrough ends with the last heir of the dynasty passing away. The user interface is a parchment-like map that can be zoomed in and out. The map covers Europe, Asia, and North Africa. The player can start with a character, ranging from e.g., a Duke in Uppland, Sweden to a Khan in the Mongol empire. CK3 tries to be as historically accurate as possible, including various government types (feudal, tribal, and clan), religions, historically relevant characters, tribes, empires, landmarks, etc. [3, 11, 15].

As common practice nowadays, Paradox Interactive has an online forum on their webpage where players can post and discuss suggestions with each other. Moreover, Paradox uses the platform "Discord," where players can chat with each other and at regular time intervals directly with the community management team and developers. Both the forum and the discord server are prominently advertised as the player launches the game. The two communication channels are also a way for users to report bugs and errors. As these bug reports would exceed the analytical focus of this study and they are usually highly technical issues, the bug reports are excluded, and the focus is placed on the online suggestion forum.

CK3, like most of Paradox Interactive's games, has a low entry barrier for both modding the game and downloading modifications to the game. Crusader Kings 3 is available on Valve's platform Steam and also enabled for the Steam Workshop. Steam Workshop is a secondary platform to Steam and gives players easy access to download modifications and for free innovators to produce them. An example of one of the most downloaded and impressive modifications is the "Lord of the Rings: Realms in Exile" modification. The free modification created by a collective of 22 modders converses the medieval setting of the game into a game with the gameplay mechanics of the base game in the setting of Middle earth. As stated earlier, despite an impressive amount and quality of user generated content in the form of modifications, this article mainly analyzes the ideas and suggestions from players on how the released game can be improved. User generated content or modifications can be seen more as a total conversion of the game, whereas the suggestions made in forums aim to improve the base game incrementally. However, I argue that both offering written suggestions/ideas and creating modified content can be categorized as free innovations, as users dedicate their free time without the aim of making a financial profit. The method applied to do so is explained in the next section.

\section{Method}

CK3 is an ideal case that shows how consumers dedicate their free time doing both, i.e., modification and sharing suggestions/ideas. The focus on the latter was chosen, as the method of a netnography was assumed to be an interesting tool to capture this vast amount of qualitative data stored in one virtual location - namely the firm's official online forum. The NVivo capture feature and word-query analysis were used for the qualitative data analysis process. Furthermore, comparing the insights gained from the online forum with the patch notes of the game makes it possible to analyze the extent to which the developers include minor and major suggestions provided by the consumers.

The technique of nethnograpy was chosen for the purpose of this study, as netnography builds upon participation and observation in publicly available forums $[16,17]$. Netnography can best be described as adapting ethnography to the online world. It is a qualitative research method that combines archival and online communication work, either through participation or observation [16]. It provides a new form of digital and network data collection. This study follows the methodology guidelines provided by Kozinets [16, 17] as well as well-cited research that used netnography within the field of interactive marketing [25], customer knowledge management [6], or similar context of video games [9, 29].

The main stages of conducting a netnography were followed according to Kozinets' guidelines [17], which are entrée, data collection, analysis, and interpretation. The initial stage of entrée means choosing the right community on which to conduct a netnography. Kozinets [16] advises to use communities with high traffic and interaction. CK3 thus was assessed as an ideal case for this study as the game enjoys a large fan base from its previous versions. Paradox Interactive published numerous 
such complex strategy games since their founding in 2004. Superb ratings and reviews prior to the release of CK3 $[3,11,15]$ also served as an indicator that the interest in this game will be enormous and the size of the community might grow further.

As an avid player of video games but unfamiliar with any game from Paradox Interactive, the author purchased CK3 shortly after its release and spent around 45 hours with the game. Besides the entertaining aspect of this familiarization with the case, this was a crucial step for data analysis as the data collected contained very specific terms and lingo from the game which outsiders might find difficult to understand (e.g., "I suggest, rather than barring a liege from educating a vassal from a realm with religious protections, put a cap on this opinion malus." [19])

For the second stage of data collection, the author followed the official online forum for CK3 on the Paradox Interactive homepage and more explicitly focused on the suggestions in the sub-forum through the period of the first four months after the game's release (September - December 2020). Data collection has been conducted by downloading a total of 1,798 forum threads from the suggestions in the sub-forum and classifying the threads with the latest version (1.3) of NVivo. NVivo is a tool for computer-based qualitative analysis. The initial post of the thread was analyzed and coded by a first generalization and a second refinement process guiding toward a theory building. Coding techniques suggested by Ryan and Bernard [24] were applied to eventually come up with the five main codes. Sub-themes were identified by applying the text and word search query features to each main theme by NVivo. The results of this data analysis - which resembles the third stage in Kozinet's [17] approach - and the fourth stage of interpretation are discussed in the findings and discussion sections of this article.

As part of using a netnographic approach, it is vital to apply a variety of methods $[17,25]$. Generally, the possibility for triangulation with other methods helps to enrich data and strengthens the generalizability of a netnography [17, 25]. For the purpose of this study, triangulation is performed by conducting interviews with active forum contributors and community managers. To do so, 20 active contributors to the CK3 suggestions online forums were contacted for an email interview. Two selection criteria were relevant for selecting participants. First, the suggestion threads have been filtered by the most views; then, as a second filter of those most viewed threads, 20 thread creators with more than 500 forum messages have been selected. The lower cap of 500 messages ensured that the participants spent a vast amount of free time discussing or making suggestions to one of Paradox Interactive's games. This selection method ensured that the members are active and thus spent a certain amount of free time making and discussing suggestions. Filtering for the most viewed threads ensured that the participants made at least one significant suggestion in the forum. Eleven users responded to an initial message, and 9 were willing to engage in a written e-mail interview after being ensured them that neither their names nor their forum usernames would be quoted in this study.

Moreover, the author joined the CK3 discord server to get in touch with the community managers. Discord is a platform that allows text, image, audio or video communication between users. The communication is moderated by community managers employed by Paradox Interactive. Those community managers also observe the official Paradox forum. One community manager and two community ambassadors have been contacted through discord, and two of them agreed to a written e-mail interview after being ensured that their real names or nicknames would not be quoted in this study.

For the sake of data triangulation, the game's patch notes and the developer's online letter to the community were assessed. This served as a basis for the data analysis as well as to get an understanding of aspects of community suggestions that the developer of CK3 has addressed and implemented post-release. Additionally, playing the game for some more hours after the release of patches 1.1 and 1.2 served to understand the changes made to the vanilla version (1.0) of the game.

The identified categories of suggestions, the insights from the interviews, and analyses of patch notes and letters to the communities are addressed in the next section.

\section{Findings}

It is common in the video game industry that games raise the most attention at the time of their release and shortly thereafter. Data from SteamDB - a statistic site on Steam, the largest digital platform where CK3 can be bought - shows that on its launch day (September 1st, 2020), the game had 97,000 users playing the game and 56,000 people watching on the streaming platform Twitch on that specific date. Four months later, on January $1^{\text {st }}$, the game had "only" 22,000 players who played the game via Steam on that day [31]. Nevertheless, according to Paradox's Q3 2020 report, the game sold over 1 million times in the first month, and one-third of the entire development cost was amortized in the first month after the release [32]. Two things are important to consider when talking about these figures. First, CK3 is also included 
in Microsoft's game subscription service "Game Pass." Thus, those users who play the game through this subscription service are not included in either the Steam users playing the game or the sold copies. Second, the previously stated Steam numbers do not account for hard-copy game sales of the game. So, the number of players that played the game on release is de facto much higher; nonetheless, the numbers from Steam serve as a good indicator.

The reason for stating these large numbers of sales in the month of the release serves as an explanation as to why the first four months after the game's release was the chosen period for this study. The enormous interest very shortly after the release of the game may also explain why a vast amount of suggestions regarding the game were made in the forum, within three weeks after the release of the game. Roughly $40 \%$ of the 1,798 forum threads that were analyzed in this sample were created between the 1st of September and the 21 st of September. The time after the release, thus, is a crucial time frame for Paradox Interactive to continuously improve and update the game. Hence, the development of the game does not stop with the release of the product. Even more, the developers must satisfy the requests from their customer base and keep the long-time motivation high so that the game is played on video streaming platforms too, which can almost be seen as digital word-to-mouth advertising. Paradox Interactive must therefore get a sense of what issues and ideas the community desires to be addressed by the developer. To ensure that the players are heard, Paradox Interactive has two community ambassadors and one community manager who were specifically dedicated to CK3. The two Community ambassadors serve as the "eye and ear" in the discussion with the community and collect all the feedback from the Paradox forum, social media (mainly Discord), and relevant influencers such as popular players who stream the game to a large audience. According to the interviewed community manager, the suggestions are in a first step mainly evaluated based on merit and feasibility. This assessment is done by the community manager who presents the collaborated and evaluated community feedback once per week to the development team, besides having more unstructured daily discussions with the development team. The team discusses potential incremental adaptations based on three criteria. These are value to current players, appeal to future players, and how well the suggestions fit the strategy for future content updates that are already planned. In comparison with other games that Paradox Interactive has released, the community manager stated that CK3 is the "bleeding edge" for their firm, when it comes to gathering community and individual player feedback. In previous games, a lot of attention was given to the community, but specifically for CK3, the firm has increased the time and resources dedicated to the community feedback.

Changing the perspective of the community members and the insights from the nine heavy contributing community members revealed a different picture. Just one of those heavy forum contributors mentioned that he was at some point in direct contact with a community manager on a suggestion that he made (which was on another Paradox Interactive game). Most of the interviewees raised some discontent that the community managers and ambassadors are too unapproachable and that they relatively seldom join their discussions. In line with that, some respondents expressed they received very little or no attention from Paradox Interactive's community team in response to the various suggestions that they made. Despite that, all the interviewees are content or point out how much they appreciate Paradox Interactive, including the community's input as well as how much effort they put into constantly updating the game incrementally, together with the new features that are added. A majority of the interviewed heavy contributors could also state at least one minor suggestion that they made to a Paradox Interactive game that was eventually included or addressed. One interviewed community member also stated that the "developer's diaries" (letter to the community that the development team post once every couple of weeks in the forum) serve as the best tool to see how the player's feedback is incorporated into the regular patches of the game. The same interviewee mentioned that in the "developer's diaries" post, it is easier to approach Paradox Interactive developers and discuss ongoing changes in upcoming updates of the game. A potential reason for this behavior from the development team perspective might be that each suggestion that is made by a player is owned by the player. So, Paradox Interactive could theoretically face copyright issues if they were to take a sophisticated suggestion made by a player and incorporate it into the game. Hence, this potentially explains the approach taken by Paradox Interactive, where they continuously post their ideas and concepts in the form of a letter to the community once the whole player feedback has been assessed.

One interviewer also pointed out a previously released game by Paradox Interactive which was "a disaster at release" in his words. The developers, however, were able to fix the game due to a large amount of community feedback and change of the project lead at Paradox Interactive. It became obvious according to the interviewee that the new community lead started to leverage on player's feedback post- 
release, which was a key to fix the game. According to the respondent's judgment, user feedback is particularly of high value if the developers "mess it up at release." CK3, however, had no such major issues at release, and the game received overwhelmingly positive reviews $[3,11]$.

Beyond the individual responses by the contributors, the netnography conducted in the forum granted more holistic insights into what players demand from the developers. Five themes emerged from the analysis, and examples for each theme will be given in this section. The themes are cross-checked with the developer's letters to the community and patch notes for the major patches 1.1 and 1.2.

Gameplay features

Suggested changes on gameplay mechanics and rules of how the game is set to play were by far the most identified requests from players based on the forum threads. There were 822 forum threads coded under this theme. A suggestion that gained a lot of attention and emerged in a lively discussion was the request to enable same-sex marriage to the game. This particular thread is the third-most viewed forum thread in the suggestion forum. The initiator of this discussion pointed out that from a gameplay perspective, this would lead to more options for marriage alliances, potentially allowing for adoption systems and in general please the LBGTQ player base of CK3. (Homosexual characters exist in the game and they can have liaisons, but marriage or adoption is not possible). The community debated on this suggestion thoroughly. Some players pointed out that this would undermine the historical adequacy of the game. In contrast, others pointed out that there are historical documents which prove that same-sex marriages and formal unions existed also in the Middle Ages. Some sort of common sense on that debate was found as one player initiated and linked to a petition where he/she suggested to Paradox Interactive to change some codes of the game software so that players could modify the game themselves. This code change would make it possible for same-sex marriage to be enabled through modifications. This new petition thread has gained a lot of support; however, up until four months past release, this feature or the changes to the code have not been included by Paradox Interactive, and the players seem to be quite unsatisfied with this. A few weeks later however, one member posted a link to an interview with a Paradox Interactive developer where he stated that they are working on making some changes to the code so at least same-sex marriage can be enabled through modification of the game.

Besides this interesting debate, the two most significant sub-themes that emerged within the "gameplay feature" theme were "warfare" and "vassalage/vassal." The rather simple warfare mechanics was one of the few negative aspects that critics pointed out in their reviews of the game. The players seem to evaluate this similarly; thus, this topic sparked a lot of suggestions from the players. In terms of the sub-theme "vassalage," which is another central feature of the game, there are a lot of minor changes included in the game based on the patch note 1.1 (87 changes) and patch note 1.2 (46 changes).

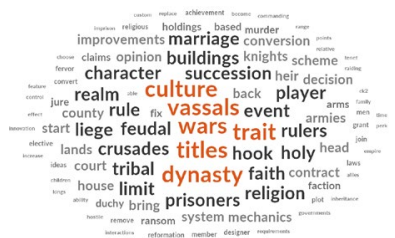

Figure 2. Word cloud for the theme "Gameplay Features"

User Interface / Quality of Life (QoL) improvements

The second main theme identified by coding the threads of the suggestions forum was User interface (UI) or Quality of Life (QoL) improvements. This theme includes suggestions made by the players which refer to improving the graphical or menu-driven interface. As the game is complex by nature, there are a lot of pop-up windows and notifications educating and informing the player on what is going on. Experienced players can turn off certain notifications and inexperienced players can hover above highlighted words in the game that opens a new window that explains certain expressions and game mechanics (Some critics ironically called the game "pop-up window simulator").

The user interface however is an aspect of the game that caused players to write a lot of suggestions on that issue. In total, 559 forum threads are coded within this category. There was a lot of discontent in the forum regarding a button that pops up at the top of the screen when there is a critical issue. Players have criticized that this button is not configurable in terms of what "critical" events cause a notification and that it cannot be completely disabled. The title of a thread that is one of the most viewed of the entire suggestion forum describes it quite drastically with a touch of sarcasm. The thread is called: "This button is the worst idea for a Paradox UI imaginable, possibly the worst idea ever to exist, even almost all civilizations that occupy our galaxy."

Other sub-themes that were identified in the players' suggestions in relation to UI or QoL improvements are "character" or "filter options." Character refers to the request from players to customize their main characters and heirs (a major feature which was introduced with patch 1.2) and "filter options," as filtering through tables with 
character stats of an opponent or a player's own dynasty is a large aspect of the game. Also, improvements on the user interface regarding "call to arms" or raising and maneuvering armies over the parchment-like map has produced a lot of suggestions from players.

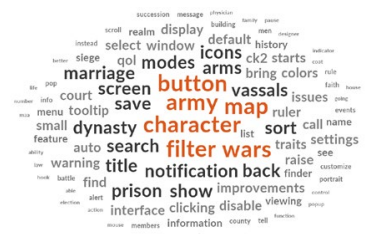

Figure 3. Word cloud for the theme "User Interface"

\section{Historical or geographical context}

Despite being an entertainment product, CK3 attempts to be as accurate as possible when it comes to the history and geography of the setting from 867 , respective, 1066 to 1459 A.D. The community also contributes to that accuracy by cross-checking facts and pointing out mistakes in the game, in terms of history and geography. A total of 213 suggestions were made in the forum on that aspect. Some of the discussions evolved around rather minor but very detailed suggestions such as increasing the diversity and number of religions to the Finno-Ugric tribes, pointing out correct medieval names of Slavic cities and discussions/suggestions on improving the Balkan region, in terms of counties and religion settings. The passion that the community shows in these aspects is quite fascinating. Most of the suggestions include long explanations, links to sources, or users drawing new maps and uploading screenshots. One of the most sophisticated suggestion was made by a user who reveals himself as a Ph.D. student of Islamic Studies and who was reviewing the game on historical accuracy in terms of Islam in $1066 \mathrm{~A}$. D. The user links to a 30-minute YouTube video where he reflects on whether the most prominent Islamic tribes in the game are correctly represented historically, according to the available historical knowledge [30].

The patch notes also reveal that Paradox Interactive is constantly making improvements on historical accuracy. A vast number of minor changes to the database can be found in both patch notes that improve the historical accuracy.

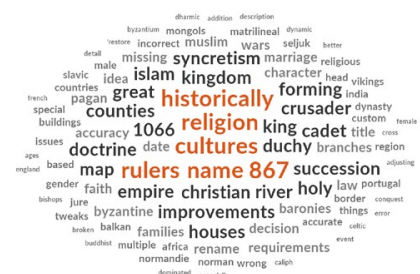

Figure 4. Word cloud for the theme "Historical \& geographical context"

\section{Artificial Intelligence}

In the forum, 110 suggestions have been coded under the theme of "Artificial Intelligence" (AI). In this context, AI stands for the behavior of the other dynasties or empires that are simulated or mimicked by the computer and competing with the player for expanding its dynasties and empire in the game. An exemplary issue for AI that was raised by a player soon after the release of the game was that "Sweden has gotten out of control" and the behavior of the Swedish and Nordic Viking tribes' expansion is too aggressive, leading in some playthroughs starting in the year 867 to spread throughout Europe and easily becoming more powerful than the Byzantine Empire. (The player ironically raised the question of whether the Swedish Tribes are buffed as Paradox Interactive is a Swedish company). This issue was addressed however in patch 1.1, where according to the patch notes one of the changes included that "Viking vassals are now more restricted when it comes to overseas conquests."

However, more threads were created on another issue, regarding what falls under the sub-theme of "Holy war AI" and "marriage." This first sub-theme "Holy War AI" mostly covered suggestions on improvements when it comes to the behavior of the allied or opponent armies in "Holy Wars." The second most prominent sub-theme in the "AI" theme was "Marriage," dealing with suggestions for improvements when it comes to suggestions on marriage to other factions. Also, herein the patch notes from patches 1.1 and 1.2 show a vast number of minor changes to the "war AI" and "marriage AI" mechanics addressing these concerns.

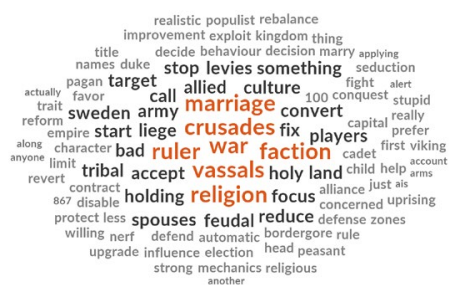

Figure 5. Word cloud for the theme "artificial intelligence"

Modding

The expression modding comes from the expression to modify. In the video game community, modding is used to create and alter software. Mods and instructions on how to mod the base game are usually shared online [23]. Not all computer games enable the community to mod their games, but Paradox Interactive actively supports users who want to mod the game with a console function that allows one to alter game characteristics, rules, and settings. Therefore, suggestions by players who are modding CK3 are also common in the suggestion forum. 
However, these were rather few (58 suggestion threads) in comparison to the other themes. Many suggestions asked about adding new console demands that would ease some modifications, and that enabling minor modifications does not block earning in-game achievements. The patch notes reveal that Paradox Interactive puts a lot of effort into satisfying the modding community and those who install mods developed from other players. This is presumably because installing mods increases the long-time motivation to play the game. Patch 1.1 included 73 minor changes that affect modding features and patch 1.2 had 31 changes.

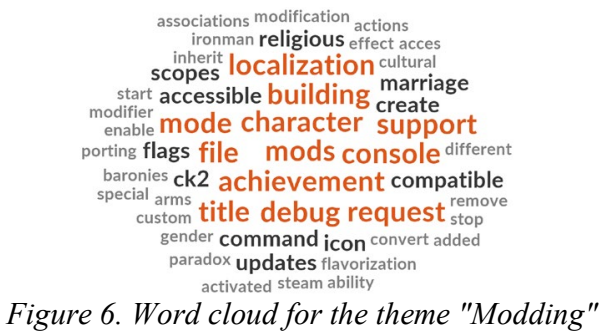

\section{Discussion}

The findings and themes elaborated in the previous section show that enthusiastic users are spending a lot of time and effort in posting their suggestions for the game. The collaborative evaluation of consumers on what could improve the current version of the game resembles a valuable source of innovation to the game developer. As stated by the community managers, the players' suggestions are addressed in weekly meetings; moreover, the patch notes indicate that many of the suggestions made by the players are implemented into updates of the game. In line with von Hippel's paradigm, the collaborative evaluation by the consumer serves as an innovation support to the producer. Paradox Interactive and other gaming firms find themselves in a pleasant situation, where they can make profits from both the free innovation paradigm and the producer paradigm. Players who diffuse free innovations in the form of modifications, like the Lord of the Ring modification for CK3, increase the attractiveness of the base game and the longevity of the game. Consequently, more consumers will be attracted to buy the base game. Moreover, feasible suggestions and ideas generated by free innovators can be picked up by the producer and included in their game through patches. This increases the quality of the game.

However, firms like Paradox Interactive have to be aware of not losing touch with their community. As many of the interviewed forum contributors have mentioned, they are often unhappy about how little their ideas are appreciated or commented on by Paradox Interactive. Whereas free innovators who release modifications on platforms like Steam Workshop, at least get a certain amount of reward through positive feedback from other players, the interviewed forum contributors, who suggest incremental but often vital changes to the game, do not seem to receive any appreciation. Triangulation with the patch notes and playing the game after the two patches have shown that many of the strongly requested and suggested changes have been addressed by the developer. Based on the insights by the community managers, forum contributions are assessed and evaluated in their weekly meetings. Therefore, these suggestions and ideas are vital for updating the game. The letters to the community by Paradox Interactive are one way to communicate more directly with the community and appreciate the free time invested by forum members. However, Paradox Interactive might serve well to incentivize ideas and suggestions contributed by their players further, which can reduce in-house $\mathrm{R} \& \mathrm{D}$ costs.

\section{Conclusion}

The main focus of this study was placed on free innovators that contribute primarily through ideas and suggestions. The case of CK3 exemplifies one of many examples for an industry where the strong interplay between consumers and producers is unique, and a community management serves as a vital idea and innovation source for developers. Success stories of user generated content that led to blockbuster hits like Dota are a good example of user innovations, but it should not overshadow the fact that a large part of consumers contribute by sharing ideas and suggestions with the producer and peers. This particular research highlights and tried to categorize the vast number of ideas that can be seen as a resource for the improvement of a product post-release. In the case of $\mathrm{CK} 3$, these suggestions ranged from the user interface to the historical accuracy of the game. The netnographic approach of this study served as a tool to understand this unique and enthusiastic user commitment. Furthermore, the categorization by themes through NVivo served as a way to examine and visualize the large number of ideas and suggestions from the community, directed at video game developers.

The video game industry is unique as it is a creative, digital, industry where the product lives on even after its release. This study has tried to capture this by showing how community members dedicate their free time to improve a producer's product, and their ideas and suggestions serve as a resource for 
incremental improvements. Moreover, by taking on a particular game as a case, this article provides a holistic overview and a visualization of the type of improvements suggested by players.

\section{References}

[1] Antorini, Y.M., and A.M. Muñiz, "The Benefits and Challenges of Collaborating with User Communities", Research-Technology Management 56(3), 2013, pp. 21-28.

[2] Bengtsson, L., "How big and important is consumer innovation in Sweden? - A comparison with five other countries", 2016, pp. 19.

[3] Borsilli, T., "Crusader Kings III Review", Strategy Gamer, 2020

https://www.strategygamer.com/reviews/crusaderkings-3/

[4] Boudreau, K.J., and L.B. Jeppesen, "Unpaid crowd complementors: The platform network effect mirage", Strategic Management Journal 36(12), 2015, pp. 17611777.

[5] Cannizzo, A., and E. Ramírez, "Towards Procedural Map and Character Generation for the MOBA Game Genre", Ingeniería y Ciencia 11(22), 2015, pp. 95-119.

[6] Chua, A.Y.K., and S. Banerjee, "Customer knowledge management via social media: the case of Starbucks", Journal of Knowledge Management 17(2), 2013, pp. 237-249.

[7] Feldman, B., "Fortnite Has Become the Instagram of Video Games", Intelligencer, 2018. https://nymag.com/intelligencer/2018/07/how-fortnitebecame-the-most-popular-video-game-on-earth.html

[8] Franke, N., E.V. Hippel, and M. Schreier, "Finding Commercially Attractive User Innovations: A Test of Lead-User Theory*", Journal of Product Innovation Management 23(4), 2006, pp. 301-315.

[9] Garcia-Alvarez, E., J. Lopez-Sintas, and A. SamperMartinez, "The Social Network Gamer's Experience of Play: A Netnography of Restaurant City on Facebook", Games and Culture, 2015.

[10] Gibbert, M., M. Leibold, and G. Probst, "Five Styles of Customer Knowledge Management, and How Smart Companies Use Them To Create Value", European Management Journal 20(5), 2002, pp. 459-469.

[11] Hafer, L., "Crusader Kings 3 Review - IGN", IGN, 2020. https://www.ign.com/articles/crusader-kings-3review

[12] von Hippel, E., Free Innovation, The MIT Press, Cambridge, MA, 2016.

[13] von Hippel, E., "Free Innovation by Consumers-How Producers Can Benefit: Consumers' free innovations represent a potentially valuable resource for industrial innovators.”, Research-Technology Management 60(1), 2017, pp. 39-42.

[14] Ho-Dac, N.N., "The value of online user generated content in product development", Journal of Business Research 112, 2020, pp. 136-146.

[15] Jackson, G., "'Crusader Kings III' Is a World of Complexity That Feels Powerfully Alive”, Vice, 2020. https://www.vice.com/en/article/jgx4b4/crusaderkings-iii-is-a-world-of-complexity-that-feelspowerfully-alive

[16] Kozinets, R., Netnography: Redefined, Sage Publications Ltd, Los Angeles, 2015.

[17] Kozinets, R.V., Netnography: The Essential Guide to Qualitative Social Media Research, SAGE Publications Ltd, 2019.

[18] Oliveira, P., and E. von Hippel, "Users as service innovators: The case of banking services", Research Policy 40(6), 2011, pp. 806-818.

[19] Paradox Interactive Forum, "Declined Guardianship Opinion Malus Cap", Paradox Interactive Forums, 2021.

https://forum.paradoxplaza.com/forum/threads/decline d-guardianship-opinion-malus-cap.1451031/

[20] Parmentier, G., and R. Gandia, "Managing Sustainable Innovation with a User Community Toolkit: The Case of the Video Game Trackmania", Creativity and Innovation Management 22(2), 2013, pp. 195-208.

[21] Parmentier, G., and V. Mangematin, "Orchestrating innovation with user communities in the creative industries", Technological Forecasting and Social Change 83, 2014, pp. 40-53.

[22] Petermeier, D., "Top 5 beliebteste eSport Spiele", ISPO, 2020. https://www.ispo.com/trends/topbeliebteste-esport-spiele

[23] Poor, N., "Computer game modders' motivations and sense of community: A mixed-methods approach", New Media \& Society 16(8), 2014, pp. 1249-1267.

[24] Ryan, G.W., and H.R. Bernard, "Techniques to Identify Themes", Field Methods 15(1), 2003, pp. 85-109.

[25] Seraj, M., "We Create, We Connect, We Respect, Therefore We Are: Intellectual, Social, and Cultural Value in Online Communities", Journal of Interactive Marketing 26(4), 2012, pp. 209-222.

[26] Steam Charts, "Dota 2 - Steam Charts", 2021. https://steamcharts.com/app/570

[27] Steam Workshop, "Steam Community:: Steam Workshop", 2021. https://steamcommunity.com/workshop/Stern

[28] The Economist, "All together now", The Economist, $2012 . \quad \mathrm{https} / / / \mathrm{www}$. economist.com/specialreport/2012/04/21/all-together-now

[29] Wang, Y.-S., W.-L. Lee, and T.-H. Hsu, "Using netnography for the study of role-playing in female online games: Interpretation of situational context model", Internet Research 27(4), 2017, pp. 905-923.

[30] YouTube, Islam DLC? PhD Designs! Crusader Kings 3 Islam and Muslim Review, 2020.

[31] "Crusader Kings III · AppID: 1158310", SteamDB. https://steamdb.info/app/1158310/

[32] "Interim report January - September 2020", Paradox Interactive - Global Games Publisher. https://www.paradoxinteractive.com/en/interimreport-january-september-2020 